\title{
Sodium and water perturbations in patients who had an acute stroke: clinical relevance and management strategies for the neurologist
}

\author{
Kevin C J Yuen (D) , ${ }^{1,2}$ Valerie Sharf, ${ }^{2,3}$ Elizabeth Smith, ${ }^{4}$ Minhwan Kim, ${ }^{5}$ \\ Alissya S M Yuen, ${ }^{6}$ Paul R MacDonald ${ }^{2,3}$
}

To cite: Yuen KCJ, Sharf $\mathrm{V}$, Smith E, et al. Sodium and water perturbations in patients who had an acute stroke: clinical relevance and management strategies for the neurologist. Stroke \& Vascular Neurology 2021;0. doi:10.1136/svn-2021001230

Received 10 July 2021 Accepted 26 November 2021

D Check for updates

(C) Author(s) (or their employer(s)) 2021. Re-use permitted under CC BY-NC. No commercial re-use. See rights and permissions. Published by BMJ.

${ }^{1}$ Departments of

Neuroendocrinology and

Neurosurgery, Barrow

Neurological Institute, Phoenix,

Arizona, USA

${ }^{2}$ University of Arizona College of Medicine and Creighton School of Medicine, Phoenix, Arizona, USA

${ }^{3}$ Department of Neurology, Barrow Neurological Institute, Phoenix, Arizona, USA

${ }^{4}$ Midwestern University, Glendale, Illinois, USA

${ }^{5}$ Creighton University School of Medicine, Phoenix, Arizona, USA

${ }^{6}$ University of California Davis, Davis, California, USA

Correspondence to Dr Kevin C J Yuen; kevin.yuen@dignityhealth.org

\section{ABSTRACT}

Sodium and water perturbations, manifesting as hyponatraemia and hypernatraemia, are common in patients who had an acute stroke, and are associated with worse outcomes and increased mortality. Other non-strokerelated causes of sodium and water perturbations in these patients include underlying comorbidities and concomitant medications. Additionally, hospitalised patients who had an acute stroke may receive excessive intravenous hypotonic solutions, have poor fluid intake due to impaired neurocognition and consciousness, may develop sepsis or are administered drugs (eg, mannitol); factors that can further alter serum sodium levels. Sodium and water perturbations can also be exacerbated by the development of endocrine consequences after an acute stroke, including secondary adrenal insufficiency, syndrome of inappropriate antidiuretic hormone secretion and diabetes insipidus. Recently, COVID-19 infection has been reported to increase the risk of development of sodium and water perturbations that may further worsen the outcomes of patients who had an acute stroke. Because there are currently no accepted consensus guidelines on the management of sodium and water perturbations in patients who had an acute stroke, we conducted a systematic review of the literature published in English and in peer-reviewed journals between January 2000 and December 2020, according to PRISMA guidelines, to assess on the current knowledge and clinical practices of this condition. In this review, we discuss the signs and symptoms of hyponatraemia and hypernatraemia, the pathogenesis of hyponatraemia and hypernatraemia, their clinical relevance, and we provide our recommendations for effective treatment strategies for the neurologist in the management of sodium and water perturbations in commonly encountered aetiologies of patients who had an acute stroke.

\section{INTRODUCTION}

Sodium and water perturbations are common in patients who had an acute stroke, affecting approximately $50 \%$ of cases. ${ }^{1}$ These disorders can lead to a wide spectrum of clinical signs and symptoms ${ }^{2}$ and are associated with increased length of hospitalisation, morbidity and mortality. ${ }^{3}{ }^{4}$ The pathophysiology is frequently misunderstood and effective management of these patients remains problematic and often sub-optimal. Currently, because there are no consensus guidelines, local guidance documents on the assessment and treatment vary in methodological rigour. Furthermore, management recommendations are not consistent, resulting in diverse, institution-based rather than evidence-based, patient-specific approaches to the management of these disorders. ${ }^{5}$

Hyponatraemia (defined as serum sodium levels $<135 \mathrm{mmol} / \mathrm{L}$ ) is frequent in patients who had an acute stroke, ${ }^{6}$ and is associated with longer hospitalisation, ${ }^{4}$ higher short-term mortality ${ }^{78}$ and poorer outcomes. ${ }^{9}{ }^{10}$ The cause of this disorder is usually due to syndrome of inappropriate antidiuretic hormone (SIADH), and less frequently, cerebral salt wasting syndrome (CSWS) and inappropriate fluid intake and loss. ${ }^{11}$ Hyponatraemia has been reported to negatively affect stroke outcomes more so when caused by CSWS than SIADH. ${ }^{12}$ Conversely, hypernatraemia (defined as serum sodium levels $>145 \mathrm{mmol} / \mathrm{L}$ ) is less common than hyponatraemia (2\% vs $10 \%$ of patients admitted from the emergency department $)^{13}$ and tends to occur when fluid replacement is insufficient to keep up with concurrent water losses. Hypernatraemia has also been shown to increase the morbidity and mortality of patients who had an acute stroke, independent of other underlying comorbidities. ${ }^{14}$ Therefore, several important management concepts are crucial to the neurologist in understanding these disorders, including body fluid compartment changes, regulation of osmolality and the need for rapid identification and effective management of these disorders. The difference between a minor and a life-threatening symptom caused by sodium perturbation disorders are often the result of the rapidity of change in serum sodium level (not the overall deficit itself), and how 
promptly the imbalance is recognised and treated. Acute stroke is a neurological emergency that requires rapid evaluation, diagnosis and treatment. In the emergency department where such patients often present, it may not be possible for the treating neurologist to obtain a detailed and accurate medical history to guide diagnostic workup and therapeutic decision making. Thus, safe and personalised, patient-specific approaches should be employed to guide decision-making in the management of these patients.

This article delineates the different types of sodium and water imbalances in patients who had an acute stroke, symptoms and signs that neurologists may encounter, pitfalls in the diagnostic workup, complications of overcorrection and provides a pragmatic approach on effective management strategies. As there are currently no consensus guidelines on management strategies of sodium and water perturbations in patients who had an acute stroke, we conducted this review process according to the preferred reporting items for systematic reviews and meta-analyses (PRISMA) guidelines. ${ }^{15}$ Four independent researchers (VS, ES, MK and ASMY) consulted PubMed articles published between January 2000 and December 2020. Their search was limited to clinical studies published in English and in peer-reviewed journals using keywords 'stroke' or 'sodium' or 'water' or 'hyponatraemia' or 'hypernatraemia' to identify studies that reflect on current knowledge and clinical practices. The selection of studies followed a screening of titles and abstracts and a full-text review of potentially eligible studies for final determination. There were 22 studies identified. Various presenting clinical scenarios were raised and management recommendations are proposed following mutual discussion and concurrent examination of relevant parts of the selected articles.

\section{PHYSIOLOGICAL REGULATION OF WATER HOMEOSTASIS}

Serum sodium levels are physiologically maintained within a narrow range, despite wide variations in water and salt intake. Serum osmolality is tightly regulated by the interaction between the secretion and action of the antidiuretic hormone vasopressin (AVP), which is synthesised in neurons of the supraoptic and paraventricular nuclei of the hypothalamus and stored in the posterior pituitary gland, and the sensation of thirst to promote water intake from the thirst centre in the hypothalamus. In a healthy adult, the average oral intake of approximately 1-3 L/day of water is required to maintain normal fluid balance and eunatraemia. ${ }^{16}$ Serum osmolality (ratio of solutes to water in the blood plasma) is the principal stimulus for AVP secretion. ${ }^{17}$ Osmoreceptors located in the hypothalamus are the major contributors of water regulation, as they are exquisitely sensitive to changes in serum osmolality. Baroreceptors located in the carotid arteries and aortic arch can also affect AVP secretion in response to decreases in blood volume or arterial pressure. After secretion, AVP binds to the V2 receptors in the

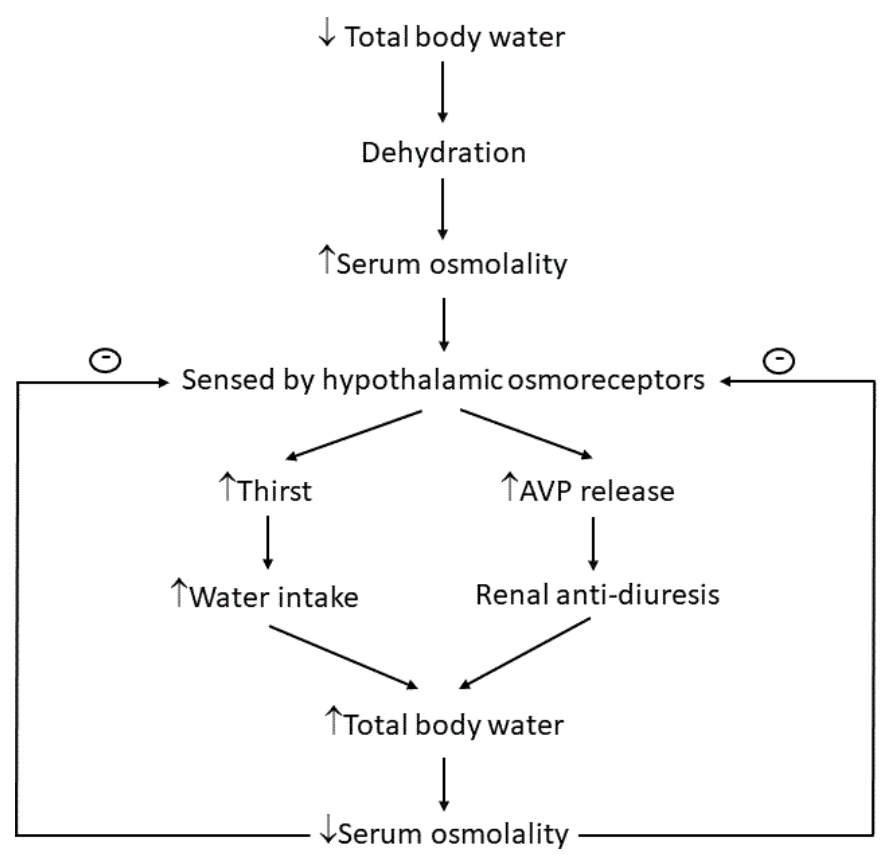

Figure 1 Physiological regulation of sodium and water homeostasis. ; inhibitory effect, $\uparrow$; increased, $\downarrow$; decreased, AVP; arginine vasopressin.

renal collecting ducts, stimulating an intracellular cascade that leads to migration of vesicle-bound aquaporin-2 to the luminal membrane of the collecting duct. ${ }^{18}$ This process renders the cells of the collecting duct permeable to water, allowing for passive water reabsorption from the kidney into the blood, such that decreased urine volume can occur, resulting in increased urine concentration. ${ }^{19}$ Simultaneously, the thirst centre in the lamina terminalis of the third ventricle is stimulated to promote water consumption. ${ }^{20}$ Thus, AVP-mediated restriction of water excretion combined with thirst-driven water intake leads to an increase in plasma water and normalisation of serum osmolality. Regulation of this homeostatic process is very intricate and efficient, resulting in serum osmolality that rarely strays by more than $2 \%$ when access to water is unrestricted. ${ }^{21}$ The mechanisms regulating sodium and water homeostasis are summarised in figure 1.

\section{PATHOGENESIS OF SODIUM AND WATER PERTURBATIONS IN PATIENTS WHO HAD A STROKE Hyponatraemia}

In acute stroke, AVP release from the posterior pituitary secretory granules is stimulated by various factors, including increased serum hyperosmolality, hypovolaemia due to decreased fluid intake, nausea, stress, pain and drugs (eg, oxcarbazepine, carbamazepine, selective serotonin reuptake inhibitors). Water retention or (less often) loss of effective solutes (sodium and potassium) in excess of water can also lead to hyponatraemia. ${ }^{22}$ Hyponatraemia caused by water retention occurs only under conditions that impair renal water excretion and does not tend to occur in patients with primary polydipsia in 
which excessive water intake can overwhelm the normal renal excretory capacity. ${ }^{22}$ The higher the plasma AVP, the more concentrated the urine. In most patients with SIADH, increasing water consumption does not adequately suppress AVP, and the urine remains concentrated. This leads to water retention, which increases total body water that subsequently lowers serum sodium levels by a dilutional effect. ${ }^{23}$ Additionally, the increase in total body water transiently expands the extracellular fluid volume causing increased urinary sodium excretion, which returns the extracellular fluid volume towards normal and further lowers serum sodium levels. Besides renal failure, primary polydipsia and low dietary solute intake, the majority of causes of hyponatraemia are associated with an absolute or relative excess of AVP (despite the presence of hypotonicity) often caused by SIADH or depletion of effective circulating intravascular volume. ${ }^{22}$

\section{Hypernatraemia}

When hypernatraemia arises in acute stroke, it is usually the result of patients not having free access to water or due to the development of diabetes insipidus (DI). Much rarer are cases of poststroke hypernatraemia caused by hypodipsia secondary damage to the thirst centre. ${ }^{24}$ If patients have free access to water, hypernatraemia is exceptional since the thirst centre is often intact after an acute stroke and is effective in preventing hypernatraemia from developing. When serum sodium levels rise, stimulation of the thirst centre and secretion of AVP occurs resulting in increased water intake and reduction in urinary water loss leading to normalisation of serum osmolality (figure 1). This maintains serum osmolality in a narrow range despite daily variations of water and sodium intake, and insensible water losses. ${ }^{25}$ If the thirst centre is damaged from the stroke, often caused by intraventricular haemorrhage, despite the secretion of AVP and the preservation of urinary concentration, hypernatraemia will develop with reductions in urine volume excretion and elevations in urine specific gravity because the patient is unable to compensate to increase free water consumption.

\section{ETIOLOGY OF HYPOTRAEMIA AND HYPERNATRAEMIA IN PATIENTS WHO HAD A STROKE}

Acute stroke can be caused by subarachnoid haemorrhage, traumatic brain injury, ischaemic and haemorrhagic stroke. The presence of underlying comorbidities (eg, diabetes mellitus, chronic renal failure, and heart failure), advanced age or concomitant medications (eg, antihypertensive agents, antidepressants and nonsteroidal anti-inflammatory drugs) are important predisposing factors to the development of hyponatraemia. ${ }^{96}$ Conversely, hypernatraemia generally rarer than hyponatraemia, and is more frequently seen in the elderly and in critically ill patients. ${ }^{27}$ Sodium and water perturbations have been prognostically linked to higher
Box 1 Aetiology of hyponatraemia in patients who had an acute stroke

\section{Conditions directly caused by stroke}

- Syndrome of inappropriate antidiuretic hormone: increased antidiuretic hormone vasopressin secretion.

- Cerebral salt wasting syndrome; inappropriate renal sodium wasting possibly due to decreased sympathetic neural input and increased brain natriuretic peptide secretion from the injured brain.

- Secondarysecondary adrenal insufficiency and/or secondary hypothyroidism: pituitary ischaemia or haemorrhage.

\section{Conditions not directly caused by stroke}

- Concurrent comorbidities: diabetes mellitus, chronic renal failure, chronic heart failure.

- Excessive administration of intravenous fluids (eg, hypotonic solutions, hypertonic solutions).

- Poor solute intake.

- Infections (eg, aspiration or hospital-acquired pneumonia).

\section{Medications}

- Antibiotics: ciprofloxacin, cotrimoxazole.

- Antiarrhythmics: amiodarone, lorcainide, propafenone.

- Antiepileptics: carbamazepine, lamotrigine, oxcarbazepine, sodium valproate, eslicarbazepine, levetiracetam, gabapentin, phenytoin, topiramate.

- Anxiolytics: benzodiazepines.

- Antidepressants: selective serotonin reuptake inhibitors, selective and norepinephrine reuptake inhibitors, tricyclic antidepressants, monoamine oxidase inhibitors.

- Antipsychotics: phenothiazines, butyrophenones, risperidone.

- Diuretics.

- Osmotic diuretic: mannitol.

- Vasopressin analogues: desmopressin, oxytocin, terlipressin, vasopressin.

- Miscellaneous: opioids, non-steroidal anti-inflammatory, nicotine, clofibrate, proton pump inhibitors, ACE inhibitors.

mortality. ${ }^{10} 28$ The aetiology of hyponatraemia and hypernatraemia in patients who had an acute stroke is summarised in boxes 1 and 2 .

\section{CLINICAL RELEVANCE}

The severity of neurological symptoms associated with hyponatraemia remains the most clinically relevant indication for prompt treatment. Symptoms of hyponatraemia can vary from mild and non-specific to severe and life-threatening, and are dependent on the level and rapidity of changes of serum sodium levels. As serum sodium levels decrease, water moves into the brain cells causing mild symptoms such as headache, fatigue, nausea, dizziness, gait disturbances, muscle cramps, falls, forgetfulness and attention deficits. The brain adapts rapidly by losing other intracellular solutes to decrease the risk of developing cerebral oedema. ${ }^{29}$ At this point, if hyponatraemia is not stabilised or corrected, patients can develop moderate to severe symptoms such as seizures, coma and/or death. Multiple case series recommend the use of hypertonic saline bolus to rapidly correct serum sodium levels, as corrections as small as $5 \mathrm{mmol} / \mathrm{L}$ can 
Box 2 Aetiology of hypernatraemia in patients who had an acute stroke

\section{Conditions directly caused by stroke}

- Diabetes insipidus: decreased antidiuretic hormone vasopressin secretion.

- Poor solute and fluid intake due to impaired neurocognition and conscious levels.

Conditions not directly caused by stroke

- Concurrent comorbidities: diabetes mellitus, chronic renal failure, chronic heart failure.

- Excessive administration of intravenous fluids (eg, hypotonic solutions, hypertonic solutions).

- Infections (eg, aspiration or hospital-acquired pneumonia).

\section{Medications}

- Water loss

- Renal losses.

- Acquired nephrogenic diabetes insipidus.

- Drug-induced hypokalaemia: diuretics, aminoglycosides, penicillin derivatives.

- Drug-induced hypercalcaemia: lithium, vitamin A or D excess.

- Other drugs: lithium, demeclocycline, colchicine, vasopres$\sin \mathrm{V}_{2}$-receptor antagonists.

- Diabetes insipidus: lithium, phenytoin, ethanol.

- Other causes: loop diuretics, osmotic diuresis, mannitol, nutritional supplementation, urea, agents that increase urea production (eg, glucocorticoids, high-protein supplements).

- Gastrointestinal losses.

- Osmotic cathartic agents: lactulose, sorbitol.

- Hypertonic sodium gain

Hypertonic sodium bicarbonate infusions.

Hypertonic sodium chloride infusions.

Hypertonic feeding preparations.

Sodium chloride-rich emetics.

Hypertonic saline enemas.

improve symptoms and achieve seizure control. ${ }^{30}$ Severe neurological symptoms such as seizures, obtundation, and coma are associated with acute hyponatraemia. ${ }^{31} \mathrm{~A}$ serum sodium drop of $>1 \mathrm{mmol} / \mathrm{L} /$ hour is associated with a higher likelihood of neurological sequelae and death, caused by cerebral oedema and increased intracranial pressure. ${ }^{32}$ Brain cells swell when water moves from the extracellular to the intracellular compartment because of a difference in effective osmolality between brain and plasma as the brain has had too little time to adapt to its hypotonic environment. Over time, the brain reduces the number of osmotically active particles within its cells (mostly potassium and organic solutes) to restore brain volume. This process takes approximately 24-48 hours, hence the rationale for using the 48-hour threshold to distinguish acute ( $<48$ hours) from chronic ( $\geq 48$ hours) hyponatraemia.

Conversely, hypernatraemia tends to develop in patients who had an acute stroke who are unable to increase fluid intake due to impaired neurocognition and conscious levels. ${ }^{24}$ As opposed to hyponatraemia, water moves out into the extracellular space resulting in brain cell shrinkage, and this can precipitate intracerebral haemorrhage caused by tearing of the cerebral vasculature. ${ }^{27}$ Symptoms of hypernatraemia include lethargy, restlessness, seizures, decreased left ventricular contractility, muscle cramps and rhabdomyolysis. The worsening progression of symptoms is not only related to absolute increases in serum sodium levels, but also the rapidity at which the increase occurs due to the speed of brain cell dehydration. $^{33}$

\section{IMPACT OF THE COVID-19 PANDEMIC ON ACUTE STROKE AND SODIUM AND WATER PERTURBATIONS}

Recent studies have shown that COVID-19 not only increases the risk of acute ischaemic strokes, ${ }^{34}{ }^{35}$ but can also cause greater severity, longer hospitalisation and higher mortality rates, ${ }^{36}$ with older patients and those with underlying cardiovascular and cerebrovascular risk factors being most vulnerable. ${ }^{34}$ The proposed mechanisms include a hypercoagulable state from systemic inflammation and cytokine storm ${ }^{37}$; postinfectious immunemediated responses; and direct viral-induced endotheliitis or endotheliopathy, potentially leading to angiopathic thrombosis. ${ }^{38}$ Additionally, sodium imbalance disorders occurs more commonly among hospitalised patients with COVID-19, with hyponatraemia being more common than hypernatraemia. ${ }^{39}$ Both of these disorders were associated with an increased hospitalisation length, and the risk of in-hospital mortality was highest in patients with moderate/severe hypernatraemia. ${ }^{39}$ Thus, when patients infected with SARS-CoV-2 present with an acute stroke and sodium and water perturbations, it can be surmised that the presentation is likely to be more severe, with a higher rate of in-hospital mortality when compared with patients without sodium and water perturbations. ${ }^{40}$ In the case of hypovolaemic hyponatraemia and hypernatraemia due to gastrointestinal fluid losses or poor fluid intake, initiation of intravenous isotonic fluid administration is recommended. In the case of hyponatraemia secondary to SIADH, fluid restriction and/or intravenous hypertonic saline infusion may be implemented, depending on the level of neurological impairment.

Currently, there are no consensus guidelines for the management of sodium and water perturbations in patients with COVID-19 presenting with an acute stroke. Therefore, because of the diversity of causes of sodium imbalance in patients with COVID-19, it is very important to establish its aetiology, as therapeutic strategies differ depending on its pathophysiological mechanism/s.

\section{ASSESSMENT AND MANAGEMENT OF HYPONATRAEMIA AND HYPERNATRAEMIA IN PATIENTS WHO HAD AN ACUTE STROKE General management principles}

The critical aspect towards the successful management of sodium and water perturbations requires a comprehensive understanding of the pathophysiological mechanism, accurate clinical assessment of the volume status 
of the patient (ie, hypovolaemia, euvolaemia or hypervolaemia), and identification of whether the hyponatraemia and hypernatraemia is acute ( $<48$ hours) or chronic ( $\geq 48$ hours) as management strategies differ. In order to establish the diagnosis and aetiology of hyponatraemia, a careful history and physical examination is required and biochemical investigations such as serum sodium, urine sodium, serum osmolality, urine osmolality, thyroid function tests and 8:00 hour serum cortisol may be needed. Management should then target towards treating the underlying aetiology and correcting the hyperosmolality or hypoosmolality.

\section{Management of hyponatraemia}

Restoration of volume depletion is imperative in the treatment of hypovolemic hyponatraemia, whereas treatment of hypervolaemic and euvolaemic hyponatraemia is more complex. In addition to correcting the underlying cause (eg, withdrawal of an offending drug), fluid restriction, administration of hypertonic solution, loop diuretics, urea and vasopressin-receptor antagonists (eg, vaptans) are amongst other therapeutic options that could be considered. Although there are clinical practice guidelines for the treatment of non-stroke hyponatraemia, currently, there is no specific reference to the treatment of hyponatraemia in patients who had an acute stroke. ${ }^{3041}$ Fluid restriction may not be appropriate in patients who had an acute stroke due to increased risk of decreased cerebral perfusion, and is contraindicated when the patient is hypovolaemic, such as hyponatraemia caused by CSWS. Additionally, isotonic saline administration should be avoided in patients who had an acute stroke with euvolaemic hyponatraemia caused by SIADH since the hyponatraemia may worsen if the urine osmolality is higher than serum osmolality. In such patients, careful administration of hypertonic $3 \%$ saline is more appropriate, especially as the overlap between SIADH and CSWS often makes it difficult to differentiate between these two conditions. The differences between these two conditions are highlighted in table 1. Conversely, in a patient who had an acute stroke who is actively seizing, a bolus of hypertonic $3 \%$ saline at a dose of $2 \mathrm{~mL} / \mathrm{kg}$ (maximum $100 \mathrm{~mL}$ ) should be administered over 10-60 min and can be repeated if seizures are still evident. ${ }^{30} \mathrm{At}$ any time during the hypertonic $3 \%$ saline infusion, the patient and the serum sodium levels must be monitored closely for any signs of deteriorating neurological status or symptoms of fluid overload.

Urea has been proposed as a potential therapeutic option as it exerts beneficial effects on cerebral oedema regardless of changes in sodium levels. ${ }^{42}$ However, clinical experience with urea administration in patients who had an acute stroke remains limited and we recommend avoiding it in hypovolaemic hyponatraemia since it induces increased renal water excretion leading to exacerbation of volume depletion and decreased cerebral perfusion.
Table 1 Differences in clinical and biochemical parameters between CSW versus SIADH

\begin{tabular}{|c|c|c|}
\hline & CSW & SIADH \\
\hline Body weight & same or $\downarrow$ & $\uparrow$ \\
\hline $\begin{array}{l}\text { Extracellular } \\
\text { volume }\end{array}$ & $\downarrow$ & $\uparrow$ \\
\hline $\begin{array}{l}\text { Signs or } \\
\text { symptoms of } \\
\text { dehydration }\end{array}$ & present & absent \\
\hline $\begin{array}{l}\text { Central venous } \\
\text { pressure }\end{array}$ & $\downarrow$ & same or $\uparrow$ \\
\hline Plasma sodium & $\downarrow$ & $\downarrow$ \\
\hline Urine sodium & $\uparrow$ & $\uparrow$ \\
\hline Net sodium loss & $\uparrow$ & normal \\
\hline Urine output & normal or $\uparrow$ & $\downarrow$ \\
\hline Serum osmolality & $\downarrow$ & $\downarrow$ \\
\hline Urine osmolality & $\uparrow$ & $\uparrow$ \\
\hline $\begin{array}{l}\text { Blood urea } \\
\text { nitrogen }\end{array}$ & normal or $\uparrow$ & $\downarrow$ \\
\hline Plasma AVP & $\downarrow$ & $\uparrow$ \\
\hline Haematocrit & normal or $\uparrow$ & $\downarrow$ \\
\hline $\begin{array}{l}\text { Plasma } \\
\text { aldosterone }\end{array}$ & $\downarrow$ & normal or $\uparrow$ \\
\hline Plasma renin & normal, $\uparrow$ or $\downarrow$ & $\downarrow$ \\
\hline Treatment & $\begin{array}{l}\text { Salt supplementation } \\
\text { and fluid replacement }\end{array}$ & Fluid restriction \\
\hline
\end{tabular}

*increased; $\downarrow$, decreased.

AVP, antidiuretic hormone vasopressin; CSW, cerebral salt wasting syndrome; SIADH, syndrome of inappropriate antidiuretic hormone.

Tolvaptan (an oral V2 receptor antagonist) is approved in the USA for the treatment of euvolaemic and hypervolaemic hyponatraemia and in Europe only for the treatment of hyponatraemia secondary to SIADH. ${ }^{43}$ On the other hand, intravenous conivaptan (a dual V1a/ V2 receptor antagonist) is approved in the USA for short-term use ( $\leq 4$ days) in hospitalised patients with non-hypovolaemic hyponatraemia. ${ }^{44}$ Vaptans are contraindicated in severe symptomatic hyponatraemia (serum sodium $<120 \mathrm{mmol} / \mathrm{L}$ ), in hypovolaemic hyponatraemia, and in patients with impaired thirst mechanisms because reductions in renal perfusion may increase the likelihood of over-rapid correction of serum sodium levels. Furthermore, studies conducted to evaluate the efficacy of vaptans in hyponatraemic patients who had an acute stroke are still lacking. Hence, use of vaptans in acute stroke should be limited only to patients with non-hypovolaemic hyponatraemia refractory to other treatment modalities, including hypertonic $3 \%$ saline.

For acute symptomatic hyponatraemia, increasing serum sodium levels by $4-6 \mathrm{mmol} / \mathrm{L}$ can decrease the risk of herniation and stop seizure activity. Larger increases should be avoided and can be harmful to patients with 
Box 3 Recommendations for management of hypornatraemia and hypernatraemia

Diagnostic approach for hyponatraemia

- In the presence of marked hyperproteinaemia or hyperlipidaemia (ie, hypertriglyceridaemia).

- 'Pseudohyponatraemia' needs to be excluded. ('Spurious' hyponatraemia refers to non-hypotonic hyponatraemia with normal values of serum osmolality (275-290 m0smol/ $\mathrm{kg})$ ).

- When osmotically active substances (eg, mannitol, glucose) are present, serum osmolality is normal or increased.

- In hyperglycaemic states, the corrected serum sodium should be calculated by increasing the measured serum sodium level by 1.4$2.4 \mathrm{mmol} / \mathrm{L}$ for every $100 \mathrm{mg} / \mathrm{dL} \mathrm{mg} / \mathrm{dL}$ of blood glucose increase above normal.

\section{Diagnostic approach for hypernatraemia}

- Prompt determination and treatment of the underlying cause: control gastrointestinal losses, fever/sepsis, hyperglycaemic, hypercalcaemia.

\section{Step-by-step management}

\section{Hyponatraemia}

- Discontinuation of the offending drugs or hypotonic solutions.

- Treatment of underlying conditions (eg, infections, hyperglycaemic, adrenal insufficiency, diabetes insipidus).

- Acute hyponatraemia (established in a period of $<48$ hours) and hyponatraemia with severe or moderate symptoms: $100-150 \mathrm{~mL}$ over 10-20 min for 2-3 times as needed or continuous infusion ( $0.25-0.5 \mathrm{~mL} / \mathrm{kg} / \mathrm{hour})$ of $3 \%$ hypertonic saline.

- Euvolaemic hyponatraemia: $3 \%$ hypertonic saline $(0.25-0.5 \mathrm{~mL} / \mathrm{kg} /$ hour) or vaptans or urea.

- Hypervolaemic hyponatraemia: $3 \%$ hypertonic saline $(0.25 \mathrm{~mL} /$ $\mathrm{kg} /$ hour $\mathrm{mL} / \mathrm{kg} /$ hour) plus intravenous furosemide $(20-40 \mathrm{mg}$ ) or vaptans.

ypovolaemic hyponatraemia: $0.9 \%$ isotonic saline.

\section{Hypernatraemia}

- Restoration to euvolaemia in hypovolaemic patients.

- The type of fluid used depends on whether there is overall fluid depletion or sodium excess.

- Only hypotonic fluids are appropriate.

- In pure water loss (eg, diabetes insipidus) use water or $5 \%$ dextrose.

- In hypotonic sodium loss (eg, gastrointestinal) use $0.18 \%$ or $0.45 \%$ sodium chloride in mild to moderate extra-cellular volume reduction states, respectively.

- The rate of hypernatraemia correction relies uponon its duration; rapid if acute and slow over 2-3 days if chronic at a maximum reduction of $10 \mathrm{mmol} / \mathrm{L} /$ day $\mathrm{mmol} / \mathrm{L} /$ day as too rapid correction is potentially dangerous.

- Add the 24-hour obligatory fluid losses and any further ongoing incidental fluid losses.

- Consider desmopressin for patients with diabetes insipidus.

- Consider anticonvulsants for patients with seizures.

\section{Important note}

Acute hyponatraemia should be promptly managed to protect from neurological sequelae, while chronic hyponatraemia should be investigated to establish aetiology and cautiously treated to avoid overcorrection.

Continued

\section{Box 3 Continued}

In acute or symptomatic hyponatraemia serum sodium levels should increase by $4-6 \mathrm{mmol} / \mathrm{L}$ within the first $4-6$ hours.

- In chronic hyponatraemia, increases in serum sodium levels should not exceed 4-6 $\mathrm{mmol} / \mathrm{L} /$ day in high risk patients and $4-8 \mathrm{mmol} / \mathrm{L} /$ day with a target maximum limit not to exceed $10-12 \mathrm{mmol} / \mathrm{L}$ in any 24 hours or $18 \mathrm{mmol} / \mathrm{L} \mathrm{mmol} / \mathrm{L}$ in any 48 hours in low risk patients of osmotic demyelination syndrome.

- In all cases (acute, symptomatic or asymptomatic hyponatraemia) the elevation in serum sodium levels should not exceed 10-12 $\mathrm{mmol} / \mathrm{L} /$ day.

Optimal management of hypernatraemia involves identifying the underlying aetiology and correcting the hypertonicity by reversing both the serum sodium and intravascular volume.

- Goal is to decrease serum sodium by $<10 \mathrm{mmol} / \mathrm{L} /$ day with close serial monitoring of serum sodium levels during the acute phase of correction.

- Seizures occurring during correction of hypernatraemia is suggestive of cerebral oedema due to rapid shifts in osmolality, and the administration of hypotonic fluids should be halted.

- The estimated free water deficit should be corrected over 48-72 hours with a decrease in serum sodium $<0.5 \mathrm{mmol} / \mathrm{L} / \mathrm{hour}$.

chronic hyponatraemia. European guidelines in nonstroke patients recommend rapid hypertonic 3\% saline infusion depending on severity of symptoms, with two 150 $\mathrm{mL}$ boluses over $20 \mathrm{~min}$ with a sodium level assessment between boluses, ${ }^{30}$ whereas US expert panels recommend $100 \mathrm{~mL}$ hypertonic 3\% saline bolus over $10 \mathrm{~min}$ and repeated as needed for symptom management in severe clinical cases. ${ }^{41}$ We recommend that the rate of correction for acute, symptomatic hyponatraemia to be $8-10 \mathrm{mmol} / \mathrm{L}$ in the first 24 hours. Serum sodium levels need to be monitored closely to minimise the risk of overcorrection (ie, no faster than 10-12 mmol/L/day for the first 24 hours), and avoid the administration of isotonic fluids and large volumes of hypertonic fluids to patients with underlying congestive heart failure or chronic renal failure (two common comorbidities in patients who had an acute stroke), as this may lead to cardiac decompensation and pulmonary oedema. In hypovolaemic hyponatraemic patients who had an acute stroke, it is imperative to avoid an overly rapid rise in serum sodium levels when volume depletion is restored as an abrupt decrease in AVP secretion and a rapid diuresis may occur. Large volumes of urine excretion at this juncture indicates an inappropriate overcorrection of hypovolaemia.

Because most hyponatraemia cases are chronic, when there is doubt of the duration of hyponatraemia, the presumption should be that the patient has chronic hyponatraemia. In this setting, large increases in serum sodium levels $(10 \mathrm{mmol} / \mathrm{L}$ in 24 hours or $18 \mathrm{mmol} / \mathrm{L}$ in 48 hours $)$ should be avoided, unless the patient is actively seizing. In patients at high risk of osmotic demyelination syndrome (eg, hypokalaemia, alcoholism, liver disease and malnutrition), correction should not exceed 4-6 mmol/L/day, whereas in patients with low risk, target maximum rise 
of 4-8 $\mathrm{mmol} / \mathrm{L} /$ day with a target maximum limit not to exceed $10-12 \mathrm{mmol} / \mathrm{L}$ in any 24 hours or $18 \mathrm{mmol} / \mathrm{L}$ in any 48 hours is recommended. ${ }^{31}$

In severe hyponatraemia cases, cerebral oedema may develop as water shifts from extracellular stores to balance out the relative hyponatraemia in the vascular space. To compensate this shift from causing too many homoeostatic changes, the brain loses osmotic solutes to try to protect itself from cerebral oedema. ${ }^{43}$ If hyponatraemia is corrected too rapidly, brain cells shrink as serum sodium is corrected. ${ }^{45}$ It has been previously established that correction of the sodium should not occur more rapidly than approximately $0.5-1 \mathrm{mmol} / \mathrm{L} /$ hour or a total of 10 to $12 \mathrm{mmol} / \mathrm{L}$ per 24 hours, while some have even proposed not to exceed $6 \mathrm{mmol} / \mathrm{L}^{46}$ Once treatment has commenced to correct the hyponatraemia, frequent serum sodium checks should be performed to allow close monitoring and adjustment of fluid infusion rates. If the serum sodium level begins to rise too rapidly and adjustment of intravenous fluids is insufficient to slow down the over rapid correction, infusion of hypotonic fluids to decrease the serum sodium level may be required to prevent the development of osmotic demyelination syndrome. This complication can be caused by exceeding generally agreed safe limits of serum sodium correction, with corrections greater than $12 \mathrm{mmol} / \mathrm{L}$ over 24 hours, $25 \mathrm{mmol} / \mathrm{L}$ over 48 hours, or inadvertent hypernatraemia during correction of hyponatraemia, ${ }^{47}$ resulting in rapid swelling of the nervous system parenchyma that cannot adapt quickly enough to the changing osmolality. Despite the severity and devastating consequences of this syndrome, neurologists should not shy away from aggressively treating symptomatic patients who had an acute stroke with hyponatraemia because without prompt treatment, seizures, coma or death may ensue.

\section{Management of hypernatraemia}

Effective management of hypernatraemia in patients who had an acute strokes requires addressing the underlying aetiology in preventing further loss of water or hypertonic sodium gain. Once eunatraemia is achieved, treatment could involve, for example, withholding loop diuretics, administering insulin in the case of hyperglycaemic, treating vomiting or diarrhoea, withholding/adjusting hypertonic tube feeds or administering desmopressin (DDAVP) for those who have developed DI. Careful history and clinical examination to determine the volume status and measurement of urine sodium and osmolality will help to ascertain the aetiology. Patients with hypovolaemia on presentation should be resuscitated with hypotonic fluids regardless of the presenting serum sodium level until vital signs are normalised. The choice of fluid replacement for hypernatraemia should be dilute relative to serum in order to replace the water deficit. This requires the estimation of the amount of water that has been lost and can be calculated by using the formula proposed by Adrogué et al. ${ }^{27}$
Water deficit $(\mathrm{L})=[$ (measured sodium $/$ normal sodium) - 1]

The choice of replacement solution to be given and infusion rate are important factors to avoid overcorrection of the hypernatraemia. Serum sodium levels should be corrected by no more than 8 to $15 \mathrm{mmol} / \mathrm{L} /$ day, which may take longer than 48 hours in patients with severe hypernatraemia $(>160 \mathrm{mmol} / \mathrm{L}){ }^{27}$ An exception is patients with acute hypernatraemia $(<48$ hours) who can be safely be corrected rapidly at a rate of $1 \mathrm{mmol} / \mathrm{L} /$ hour. As in management of hyponatraemia, frequent measurement of serum sodium levels is critical for monitoring the response to therapy and adjusting the rate or choice of intravenous fluid. Except for hypovolaemic patients requiring resuscitation with isotonic $0.9 \%$ saline, all other patients should receive either half $0.45 \%$ saline or $5 \%$ dextrose water infusions to replace the water deficit and ongoing fluid losses.

Patients with hypervolaemic hypernatraemia present a different therapeutic challenge as the volume expansion in these patients inhibits the release of AVP, thereby promoting water excretion in the urine. Cessation of the inciting factors and administration of water is usually the initial step. Treatment with loop diuretics would enhance more aquaresis than natriuresis, thereby exacerbating the hypertonicity. Infusing dextrose $5 \%$ water would address the hypertonicity, but might worsen the volume overload state. Simultaneous use of intravenous dextrose $5 \%$ water and loop diuretics can be used to lower the serum sodium in addition to achieving a net negative total body water balance. Rarely, haemodialysis has been used, especially in cases where hydration with or without diuresis have failed to bring the sodium level down to the desired target or in patients with worsening renal function in whom there are indications for renal replacement therapy. ${ }^{46}$

For patients with DI, DDAVP treatment can be administered orally, intranasally, subcutaneously or intravenously. Thiazide diuretics rarely can be considered as it decreases urine volume and increase urine osmolality. The mechanism of the paradoxical antidiuretic effect of thiazides in DI is thought to be related to volume contraction, leading to an increase in proximal tubular reabsorption of water and sodium thereby decreasing distal delivery of water and subsequent excretion.

Despite the paucity of data, hyperosmolar agents (eg, mannitol and hypertonic 3\% saline) are still used by some clinicians in patients who had an acute stroke to induce hypernatraemia in order to decrease raised intracranial pressure and to prevent cerebral oedema. ${ }^{48} 49$ As there are currently no accepted consensus guidelines regarding optimal serum sodium levels in patients who had an acute stroke, we recommend that hyperosmolar agents be used cautiously in bolus form only to selected patients (those without risk factors, eg, alcoholism, liver disease, renal disease and previous strokes that would suggest a poorer prognosis) and closely monitor serum sodium levels targeting levels to not exceed $145 \mathrm{mmol} / \mathrm{L}$. Box 3 summarises our recommendations for management of 
hyponatraemia and hypernatraemia in patients who had an acute stroke.

\section{CONCLUSIONS}

Sodium and water perturbations are common in patients who had an acute stroke, with signs and symptoms that can range from asymptomatic to severe neurological manifestations such as seizures, cerebral oedema, coma or even death. Infection with COVID-19 can further increase the risk of negative outcomes in these patients presenting with concurrent sodium and water perturbations. Despite the lack of consensus guidelines for the management of sodium and water perturbations in patients who had an acute stroke, neurologists need to be well versed in identifying the different aetiologies of hyponatraemia and hypernatraemia in order to implement an effective management strategy to ensure optimal outcomes for these patients. Sodium and water perturbations in patients who had a stroke that present acutely require emergent intervention but only after any intravascular volume losses have been corrected first, often best achieved by isotonic saline infusion. As hyponatraemia is typically caused by a defect in water excretion, and hypernatraemia by water deficit or a defect in thirst regulation, chronic sodium perturbations ( $>48$ hours) should only be corrected in approximately the same time frame as it initially occurred because if corrected too rapidly, serious neurological consequences may ensue. Neurologists should collaborate with endocrinologists, especially when the sodium and water perturbations are complex and difficult to correct.

Contributors KCJY conceptualised, wrote and edited the manuscript; VS, ES, MK and ASMY performed literature search; VS, ES, MK, ASMY and PRM reviewed, critiqued and edited the manuscript. All authors have read and agreed to the submitted and published version of the manuscript.

Funding The authors did not receive any funding for the preparation of this manuscript.

Competing interests None declared.

Patient consent for publication Not applicable.

Provenance and peer review Commissioned; externally peer reviewed.

Open access This is an open access article distributed in accordance with the Creative Commons Attribution Non Commercial (CC BY-NC 4.0) license, which permits others to distribute, remix, adapt, build upon this work non-commercially, and license their derivative works on different terms, provided the original work is properly cited, appropriate credit is given, any changes made indicated, and the use is non-commercial. See: http://creativecommons.org/licenses/by-nc/4.0/.

ORCID iD

Kevin C J Yuen http://orcid.org/0000-0002-8169-2728

\section{REFERENCES}

1 El-Fawal BM, Badry R, Abbas WA, et al. Stress hyperglycemia and electrolytes disturbance in patients with acute cerebrovascular stroke. Egypt J Neurol Psychiatr Neurosurg 2019;55:86.

2 Ehtesham M, Mohmand M, Raj K, et al. Clinical spectrum of hyponatremia in patients with stroke. Cureus 2019;11:e5310.

3 Gala-Błądzińska A, Czarnota J, Kaczorowski R, et al. Mild hyponatremia discovered within the first 24 hours of ischemic stroke is a risk factor for early post stroke mortality. Adv Clin Exp Med 2019;28:1321-7.
4 Shima S, Niimi Y, Moteki Y, et al. Prognostic significance of hyponatremia in acute stroke: a systematic review and metaanalysis. Cerebrovasc Dis 2020;49:531-9.

5 Brennan M, Mulkerrin L, Wall D, et al. Suboptimal management of hypernatraemia in acute medical admissions. Age Ageing 2021;50:990-5.

6 Liamis G, Rodenburg EM, Hofman A, et al. Electrolyte disorders in community subjects: prevalence and risk factors. Am J Med 2013;126:256-63.

7 Chen Z, Jia Q, Liu C. Association of hyponatremia and risk of shortand long-term mortality in patients with stroke: a systematic review and meta-analysis. J Stroke Cerebrovasc Dis 2019;28:1674-83.

8 Shah A, Sabir S, Artani M, et al. Significance of hyponatremia as an independent factor in predicting short-term mortality in patients with hemorrhagic stroke. Cureus 2019;11:e4549.

9 Rodrigues B, Staff I, Fortunato G, et al. Hyponatremia in the prognosis of acute ischemic stroke. J Stroke Cerebrovasc Dis 2014;23:850-4.

10 Soiza RL, Cumming K, Clark AB, et al. Hyponatremia predicts mortality after stroke. Int J Stroke 2015;10 Suppl A100:50-5.

11 Mahesar SA, Memon SF, Mustafa S, et al. Evaluation of hyponatremia in ischemic stroke patients in a tertiary care hospital of Karachi, Pakistan. Cureus 2019;11:e3926.

12 Saleem S, Yousuf I, Gul A, et al. Hyponatremia in stroke. Ann Indian Acad Neurol 2014;17:55-7.

13 Arampatzis S, Frauchiger B, Fiedler G-M, et al. Characteristics, symptoms, and outcome of severe dysnatremias present on hospital admission. Am J Med 2012;125:1125 e1121-5.

14 Vedantam A, Robertson CS, Gopinath SP. Morbidity and mortality associated with hypernatremia in patients with severe traumatic brain injury. Neurosurg Focus 2017;43:E2.

15 Liberati A, Altman DG, Tetzlaff J, et al. The PRISMA statement for reporting systematic reviews and meta-analyses of studies that evaluate health care interventions: explanation and elaboration. Ann Intern Med 2009;151:W65-94.

16 Kaplan LJ, Kellum JA. Fluids, pH, ions and electrolytes. Curr Opin Crit Care 2010;16:323-31.

17 Robertson GL. Antidiuretic hormone. normal and disordered function. Endocrinol Metab Clin North Am 2001;30:671-94. vii.

18 Robben JH, Knoers NVAM, Deen PMT. Regulation of the vasopressin V2 receptor by vasopressin in polarized renal collecting duct cells. Mol Biol Cell 2004;15:5693-9.

19 Loh JA, Verbalis JG. Disorders of water and salt metabolism associated with pituitary disease. Endocrinol Metab Clin North Am 2008;37:213-34. X.

20 McKinley MJ, Johnson AK. The physiological regulation of thirst and fluid intake. News Physiol Sci 2004;19:1-6.

21 Robertson GL, Aycinena P, Zerbe RL. Neurogenic disorders of osmoregulation. Am J Med 1982;72:339-53.

22 Liamis G, Milionis HJ, Elisaf M. Hyponatremia in patients with infectious diseases. $J$ Infect 2011;63:327-35.

23 Rondon-Berrios H, Agaba El, Tzamaloukas AH. Hyponatremia: pathophysiology, classification, manifestations and management. Int Urol Nephrol 2014;46:2153-65.

24 Ramthun M, Mocelin AJ, Alvares Delfino VD. Hypernatremia secondary to post-stroke hypodipsia: just add water! NDT Plus 2011;4:236-7.

25 Fabris A, Ronco C, Pellanda MV, et al. Fluctuation of vasopressin secretion in chronic hypernatremia. Nephron 1990;54:245-8.

26 Huang W-Y, Weng W-C, Peng T-I, et al. Association of hyponatremia in acute stroke stage with three-year mortality in patients with firstever ischemic stroke. Cerebrovasc Dis 2012;34:55-62.

27 Adrogué HJ, Madias NE. Hyponatremia. N Engl J Med 2000;342:1581-9.

28 Qureshi Al, Suri MFK, Sung GY, et al. Prognostic significance of hypernatremia and hyponatremia among patients with aneurysmal subarachnoid hemorrhage. Neurosurgery 2002;50:749-55. discussion 755-746.

29 Arieff Al, Llach F, Massry SG. Neurological manifestations and morbidity of hyponatremia: correlation with brain water and electrolytes. Medicine 1976;55:121-9.

30 Spasovski G, Vanholder R, Allolio B, et al. Clinical practice guideline on diagnosis and treatment of hyponatraemia. Eur J Endocrinol 2014;170:G1-47.

31 Sterns RH. Severe symptomatic hyponatremia: treatment and outcome. A study of 64 cases. Ann Intern Med 1987;107:656-64

32 Chawla A, Sterns RH, Nigwekar SU, et al. Mortality and serum sodium: do patients die from or with hyponatremia? Clin J Am Soc Nephrol 2011;6:960-5.

33 Aiyagari $\mathrm{V}$, Deibert E, Diringer MN. Hypernatremia in the neurologic intensive care unit: how high is too high? J Crit Care 2006;21:163-72. 
34 Li Y, Li M, Wang M, et al. Acute cerebrovascular disease following COVID-19: a single center, retrospective, observational study. Stroke Vasc Neurol 2020;5:279-84.

35 Tu TM, Seet CYH, Koh JS, et al. Acute ischemic stroke during the convalescent phase of asymptomatic COVID-2019 infection in men. JAMA Netw Open 2021;4:e217498.

36 Topcuoglu MA, Pektezel MY, Oge DD, et al. Stroke mechanism in COVID-19 infection: a prospective case-control study. J Stroke Cerebrovasc Dis 2021;30:105919.

37 Ellul MA, Benjamin L, Singh B, et al. Neurological associations of COVID-19. Lancet Neurol 2020;19:767-83.

38 Varga Z, Flammer AJ, Steiger P, et al. Endothelial cell infection and endotheliitis in COVID-19. Lancet 2020;395:1417-8.

39 Hirsch JS, Uppal NN, Sharma P, et al. Prevalence and outcomes of hyponatremia and hypernatremia in patients hospitalized with COVID-19. Nephrol Dial Transplant 2021;36:1135-8.

40 Bass DI, Meyer RM, Barros G, et al. The impact of the COVID-19 pandemic on cerebrovascular disease. Semin Vasc Surg 2021;34:20-7.

41 Verbalis JG, Goldsmith SR, Greenberg A, et al. Diagnosis, evaluation, and treatment of hyponatremia: expert panel recommendations. Am J Med 2013;126:S1-42.
42 Annoni F, Fontana V, Brimioulle S, et al. Early effects of enteral urea on intracranial pressure in patients with acute brain injury and hyponatremia. J Neurosurg Anesthesiol 2017;29:400-5.

43 Schrier RW, Gross P, Gheorghiade M, et al. Tolvaptan, a selective ora vasopressin V2-receptor antagonist, for hyponatremia. N Engl J Med 2006;355:2099-112

44 Zeynalov E, Jones SM, Seo J-W, et al. Arginine-Vasopressin receptor blocker conivaptan reduces brain edema and blood-brain barrier disruption after experimental stroke in mice. PLoS One 2015;10:e0136121.

45 Kovacs L, Robertson GL. Disorders of water balance--hyponatraemia and hypernatraemia. Baillieres Clin Endocrinol Metab 1992;6:107-27.

46 Harring TR, Deal NS, Kuo DC. Disorders of sodium and water balance. Emerg Med Clin North Am 2014;32:379-401.

47 Sterns $\mathrm{RH}$, Riggs JE, Schochet SS. Osmotic demyelination syndrome following correction of hyponatremia. N Engl J Med 1986;314:1535-42.

48 Ryu JH, Walcott BP, Kahle KT, et al. Induced and sustained hypernatremia for the prevention and treatment of cerebral edema following brain injury. Neurocrit Care 2013;19:222-31.

49 Schwarz S, Georgiadis D, Aschoff A, et al. Effects of hypertonic $(10 \%)$ saline in patients with raised intracranial pressure after stroke. Stroke 2002;33:136-40. 